\title{
Wilf classes of pairs of permutations of length 4
}

\author{
Ian Le* \\ Mathematics Department \\ Harvard University \\ 1 Oxford St., Cambridge, MA 02138
}

Submitted: Nov 14, 2004; Accepted: Apr 24, 2005; Published: May 26, 2005

Mathematics Subject Classifications: 05A05, 05A15

\begin{abstract}
$S_{n}\left(\pi_{1}, \pi_{2}, \ldots, \pi_{r}\right)$ denotes the set of permutations of length $n$ that have no subsequence with the same order relations as any of the $\pi_{i}$. In this paper we show that $\left|S_{n}(1342,2143)\right|=\left|S_{n}(3142,2341)\right|$ and $\left|S_{n}(1342,3124)\right|=\left|S_{n}(1243,2134)\right|$. These two facts complete the classification of Wilf-equivalence classes for pairs of permutations of length four. In both instances we exhibit bijections between the sets using the idea of a "block", and in the former we find a generating function for $\left|S_{n}(1342,2143)\right|$.
\end{abstract}

\section{Introduction}

Let $S_{n}$ denote the symmetric group of permutations of $1,2, \ldots, n$. We say that a permutation $\sigma \in S_{n}$ contains another permutation $\pi \in S_{m}(m \leq n)$ if there exists $i_{1}, i_{2}, \ldots, i_{m}$ with $i_{1}<i_{2}<\cdots<i_{m}$ such that

$$
\sigma\left(i_{k}\right)<\sigma\left(i_{l}\right) \quad \text { if and only if } \quad \pi(k)<\pi(l)
$$

for all $k$ and $l$, i.e., the subsequence $\sigma\left(i_{1}\right), \sigma\left(i_{2}\right), \ldots, \sigma\left(i_{m}\right)$ has the same order relations as $\pi(1), \pi(2), \ldots, \pi(m)$. For example, the sequence 3167452 contains the permutation 132 (shown in bold), while 42531 does not contain 123. We sometimes say that $\sigma\left(i_{1}\right), \sigma\left(i_{2}\right), \ldots, \sigma\left(i_{m}\right)$ is an "occurrence of $\pi$ (in $\sigma$ )", or that $\sigma\left(i_{1}\right), \sigma\left(i_{2}\right), \ldots, \sigma\left(i_{m}\right)$ is a " $\pi$ subsequence" of $\sigma$.

We say that $\sigma$ avoids $\pi$ if $\sigma$ contains no occurrences of $\pi$. For example, 42531 avoids 123 and 32154687 avoids both 231 and 312. We denote the set of permutations in $S_{n}$ that avoid $\pi_{1}, \pi_{2}, \ldots, \pi_{r}$ by $S_{n}\left(\pi_{1}, \pi_{2}, \ldots, \pi_{r}\right)$. A problem of much interest is to determine

*Please send correspondence to the following address: 67 Danville Dr., Princeton Jct., NJ 08550 
$\left|S_{n}\left(\pi_{1}, \pi_{2}, \ldots, \pi_{r}\right)\right|$, the number of elements in $S_{n}\left(\pi_{1}, \pi_{2}, \ldots, \pi_{r}\right)$. For example, a result of Erdös-Szekeres tells us that $S_{n}(123 \cdots k, l \cdots 321)=0$ for $n \geq k l-k-l$. Also, it is well known that $S_{n}(132)=\frac{1}{n+1}\left(\begin{array}{c}2 n \\ n\end{array}\right)=C_{n}$, the $n^{t h}$ Catalan number [5]. Another problem is to determine when, for some two permutations $\pi_{1}, \pi_{2} \in S_{m},\left|S_{n}\left(\pi_{1}\right)\right|=\left|S_{n}\left(\pi_{2}\right)\right|$ for all $n$. In this case, we say that $\pi_{1}$ and $\pi_{2}$ are Wilf-equivalent. We may also investigate when sets of permutations are Wilf-equivalent: we say that $\left\{\pi_{1}, \pi_{2}, \ldots, \pi_{r}\right\}$ and $\left\{\tau_{1}, \tau_{2}, \ldots, \tau_{s}\right\}$ are Wilf-equivalent if $\left|S_{n}\left(\pi_{1}, \pi_{2}, \ldots, \pi_{r}\right)\right|=\left|S_{n}\left(\tau_{1}, \tau_{2}, \ldots, \tau_{r}\right)\right|$ for all $n$. We usually consider the case where $r=s$ and $\pi_{i}$ and $\tau_{i}$ are of the same length for each $i$.

If we view the permutation $\sigma \in S_{n}$ as a matrix in the standard way, we see that $\sigma$ contains $\pi \in S_{m}$ exactly when the matrix of $\sigma$ contains the matrix of $\pi$ as a submatrix. From this it is clear that the (up to eight) permutations obtained from $\pi$ by the symmetries of the dihedral group of order eight are Wilf-equivalent. These symmetries correspond to reversing $\pi$ (a reflection about the vertical axis), switching $i$ and $m+1-i$ for $1 \leq i \leq m$ (a reflection about the horizontal axis), taking the inverse of $\pi$ (a reflection across the upper left-lower right diagonal), and combinations of these transformations. Similarly, given a set of permutations, applying any of these symmetries to all of them gives us sets of permutations that are Wilf-equivalent to the original set. The main task in putting sets of permutations into Wilf classes is to find the equivalences that do not arise from these symmetries. For example, the Wilf classes for single permutations of length 4, 5, 6, and 7 are known.

There are, up to symmetry, 56 classes of pairs of permutations of length four. Numerical data in [4] shows that there are only five possible cases of Wilf-equivalences among these pairs. Three of these were in fact shown to be Wilf-equivalences([4], [6], [1], [2], $[3],[7])$, and in this paper we show that the remaining two cases are also cases of Wilfequivalence. In particular, the pair $\{1342,2143\}$ is Wilf-equivalent to $\{3142,2341\}$ and $\{1342,3124\}$ is Wilf-equivalent to $\{1243,2143\}$.

\section{Pairs of Permutations of Length 4}

In this section we will prove our main results:

Theorem $1\left|S_{n}(1342,2143)\right|=\left|S_{n}(3142,2341)\right|$.

Moreover, if we let $c_{n}=\left|S_{n}(1342,2143)\right|$, then

$$
\sum_{n=0}^{\infty} c_{n} x^{n}=\frac{1-\sqrt{1-8 x+16 x^{2}-8 x^{3}}}{4 x(1-x)} .
$$

Theorem $2\left|S_{n}(1342,3124)\right|=\left|S_{n}(1243,2134)\right|$.

Our main approach will be to analyze the block structure of permutations, an approach originally used in [6]. In the first case, we will find that the permutations in $S_{n}(1342,2143)$ and $S_{n}(3142,2341)$ will in general have four blocks, and that the block structures in each of these sets are very similar. Moreover, we will be able to obtain an explicit bijection 


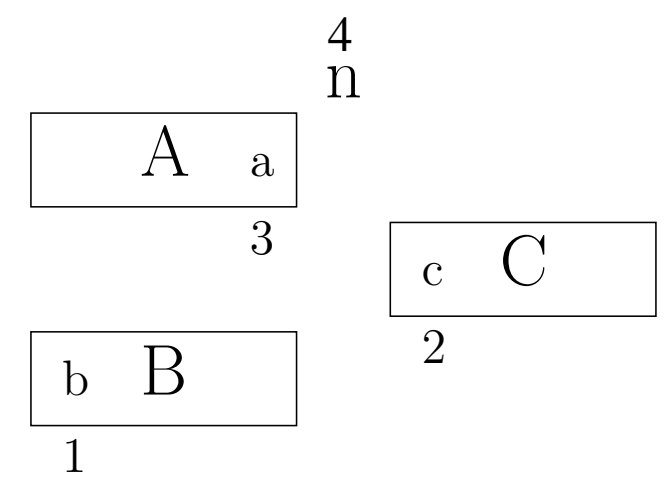

Figure 1: banc is an occurrence of 1342

between these sets and find a recurrence and generating function for $S_{n}(1342,2143)$. In the second case, we will find that permutations in $S_{n}(1342,3124)$ and $S_{n}(1243,2134)$ will generally have similar block structures, with a decreasing series of blocks on both sides of $n$. This will again allow us to exhibit a bijection.

For $\sigma \in S_{n}$, a block is a maximal subsequence of consecutive integers occurring on one side of $n$. For example, in the permutation $62111278134103519 \in S_{13}$, the blocks

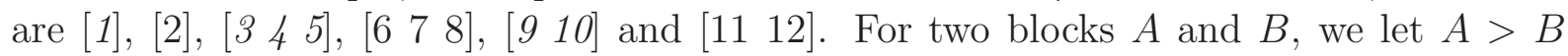
mean that every number in $A$ is greater than every number in $B$.

\subsection{Permutations in $S_{n}(1342,2143)$}

Proof of Theorem 1: We claim that any permutation $\sigma \in S_{n}(1342,2143)$ has two or fewer blocks to the left of $n$. First consider any two distinct blocks $A$ and $B$ to the left of $n$. Without loss of generality, $A>B$. Then because $A$ and $B$ are distinct blocks, there is a block $C$ to the right of $n$ such that $B<C<A$. Now if any element $b \in B$ occurred to the left of an element $a \in A$ [Figure 1], then we would have the subsequence banc contained in $\sigma$. But $b<c<a<n$, so that banc would be an occurrence of 1342 . Thus all numbers in the block $B$ lie to the right of all the numbers in block $A$. Since $A$ and $B$ could be any two blocks to the left of $n$, all the blocks on the left occur in decreasing order.

Now suppose there were three or more blocks on the left hand side. Let $A_{1}, A_{2}, A_{3}$ be three of these blocks, with $A_{1}>A_{2}>A_{3}$ so that they occur in the order $A_{1}, A_{2}, A_{3}$ [Figure 2]. Then because $A_{1}$ and $A_{2}$ are distinct, there is some block $B$ to the right of $n$ between them, i.e., $A_{2}<B<A_{1}$. Then if we have $a_{2} \in A_{2}, a_{3} \in A_{3}$, and $b \in B$, then $a_{3}<a_{2}<b<n$, so that the subsequence $a_{2} a_{3} n b$ is an occurrence of 2143 . Thus there are two or fewer blocks on the left side of $n$, and they are in decreasing order.

We now proceed to count the number of permutations $\sigma$ in $S_{n}(1342,2143)$. We break this up into three cases.

Case 1: There are two blocks $A>B$ on the left of $n$.

There is no element $x$ on the right-hand side of $n$ such that $x>A$. For if there were, 

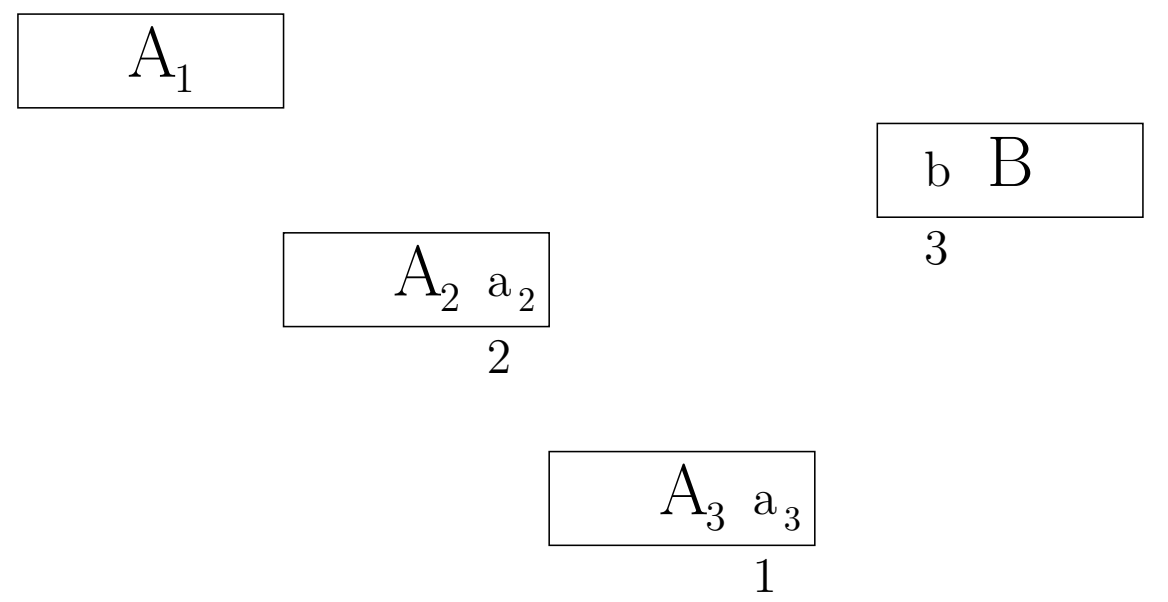

Figure 2: $a_{2} a_{3} n b$ is an occurrence of 2143

taking $a \in A$ and $b \in B$, we would have that $a b n x$ is an occurrence of 2143 . Thus on the right side of $n$ there are at most two blocks: there must be a block $C$ with $B<C<A$ and there is possibly a block $D$ with $D<B$. Moreover, the numbers in the block $B$ must occur in increasing order, for if $B$ contained a decreasing subsequence $b b^{\prime}$, then, taking $c \in C$, we would have that $b b^{\prime} n c$ is an occurrence of 2143 [Figure 3]. Let $\tilde{b} \in B$ be the the largest element of $B$. Now clearly the block $A$ must avoid 1342 and 2143, and the subsequence of $\sigma$ formed by $\tilde{b}, C$, and $D$ must avoid 1342 and 2143 . We claim that this is a sufficient condition for $\sigma$ to avoid 1342 and 2143.

Proposition 3 In the notation above, if the elements of $B$ occur in increasing order, $A$ avoids 1342 and 2143, and the subsequence of $\sigma$ formed by $\tilde{b}, C$, and $D$ avoids 1342 and 2143, then the permutation $\sigma$ avoids 1342 and 2143.

Proof of Proposition 3: Let $\sigma$ satisfy the above conditions [Figure 4a]. Suppose there were a 1342 subsequence with $n$ acting as the " 4 ". Then the 1 would have to be in either block $A$ or block $B$. If it were in block $A$, then the 3 (being larger than the 1 ) would have to be in block $A$ as well. Because $A$ is a block of consecutive numbers, the 2 would have to lie in $A$ as well, but the 2 needs to lie after $n$ in $\sigma$. If the 1 were in block $B$, the 3 (occurring after the 1 ) would also have to occur in block $B$. But that would force the 2 to occur in block $B$, again a contradiction because the 2 must occur after the 4 . Now suppose that the 4 occurred in block $A$. This would force the 1 and the 3 to lie block $A$ as well, which would then force the 2 to lie in this block, contradicting the assumption that the block $A$ avoids 1342. If the 4 occurred in the block $B$, the 1 and the 3 , being smaller, must also occur in block $B$. Again, this forces the 2 to occur in block $B$, but $B$ avoids 1342 because it is strictly increasing. If the 4 occurred in block $D$, then because $D$ is the smallest block, we would have 1342 occurring in entirely inside $D$, contradicting 


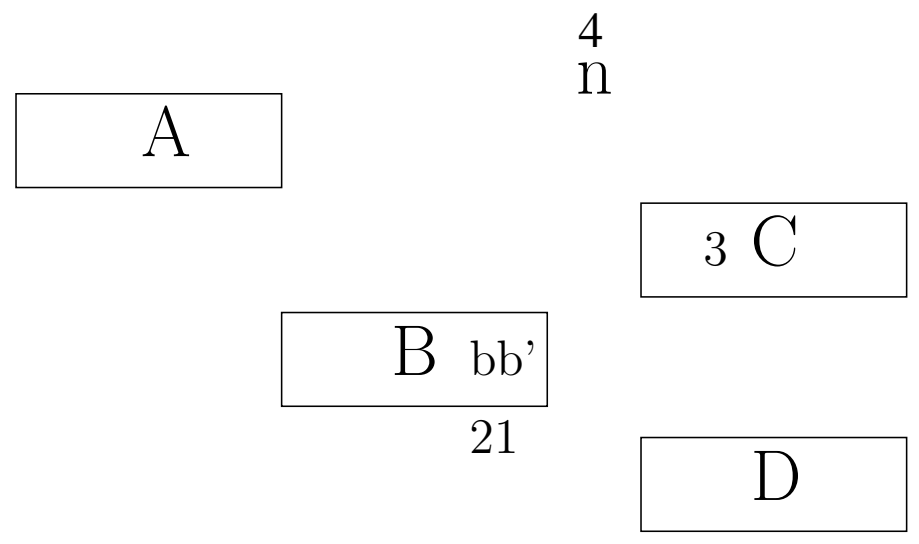

Figure 3: $b b^{\prime} n c$ is an occurrence of 2143

n

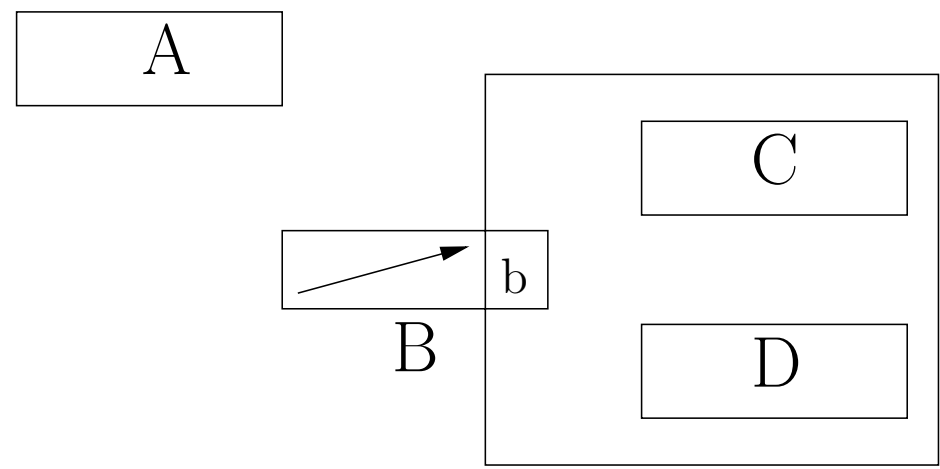

Figure 4a: $A$ avoids 1342 and 2143 and $\tilde{b}, C$ and $D$ avoids 1342 and 2143 
the assumption that the subsequence formed by $\tilde{b}, C$, and $D$ avoids 1342 . Finally, if the 4 were to occur in block $C$, the 1 and the 3 could not both occur in block $B$, otherwise the 2 would be forced to occur in block $B$ as well. Thus any 1342 subsequence with a 4 in $C$ can contain at most one member of $B$. We can take this member to be $\tilde{b}$, but this again contradicts the assumption that the subsequence formed by $\tilde{b}, C$ and $D$ avoids 1342 .

Now suppose there were a 2143 subsequence in $\sigma$. If $n$ was the 4 , then the 2 could not lie in $A$, because there would be nothing on the right side of $n$ large enough to act as a 3. But if the 2 occurred in block $B$, then the 1 would have to occur there as well, but it cannot because $B$ is strictly increasing by assumption. If the 4 occurred in block $A$, then the 2 and the 1 would have to occur in block $A$ as well, forcing the 3 to occur there, contrary to the assumption that $A$ avoids 2143 . If the 4 were in $C$, then, as before, the 2 and the 1 can't both be in $B$, so that only the 2 can occur in $B$. But then we may also take $\tilde{b}$ to be the 2 , in which case we have a contradiction because the subsequence formed by $\tilde{b}, C$ and $D$ avoids 2143. Finally, if the 4 occurs in either $B$ or $D$, the entire 2143 subsequence will lie in either $B$ or $D$, respectively. In the former case, we get a contradiction because $B$ is increasing, and in the latter because the subsequence $\widetilde{b}, C$, and $D$ avoids 2143. Thus there can be no occurrences of 1342 or 2143 for $\sigma$ satisfying the conditions of the proposition.

We may now count the number of permutations in $S_{n}(1342,2143)$ with two blocks on the left. Let $\left|S_{n}(1342,2143)\right|=c_{n}$. Let $A$ have length $i$. Then if $B$ has length $j$, the subsequence formed by $\tilde{b}, C$ and $D$ has length $n-i-j$. But $\tilde{b}$ cannot be the largest number in this subsequence, because the block $C$ of numbers on the right of $n$ larger than $\tilde{b}$ must be non-empty. Consider a permutation $\tau \in S_{k}(1342,2143)$. Then the permutation $\tau^{\prime}$ formed by adding $k+1$ to the left of $\tau$ still avoids 1342 and 2143, because the $k+1$ can only act as a 4 , and it can't do so as the left-most number in the permutation. Thus there are $c_{k}$ permutations in $S_{k+1}(1342,2143)$ that begin with $k+1$, where $c_{k}=\left|S_{n}(1342,2143)\right|$, and there are $c_{k+1}-c_{k}$ permutations in $S_{k+1}(1342,2143)$ where the first number is not the largest. So there are $c_{n-i-j}-c_{n-i-j-1}$ possibilities for the subsequence formed by $\tilde{b}$, $C$, and $D$. Summing over $j$ (where we sum $j<n-i-1$ because the block $C$ must be non-empty), we get

$$
\sum_{j=1}^{n-i-2} c_{n-i-j}-c_{n-i-j-1}=c_{n-i-1}-c_{1}=c_{n-i-1}-1 .
$$

Then multiplying by $c_{i}$ and summing over $i$ (we include $i=n-1, n-2$ for convenience, as in these cases $\left.c_{n-i-1}-1=0\right)$, we get

$$
\sum_{i=1}^{n-1} c_{i}\left(c_{n-i-1}-1\right)
$$

Case 2: There is one block to the left of $n$, and there is a larger block to the right of $n$.

This case reduces to Case 1 with the length of block $A$ equal to zero. Letting $B$ be the block on the left of $n, C$ be the larger block on the right, and $D$ the possibly empty 


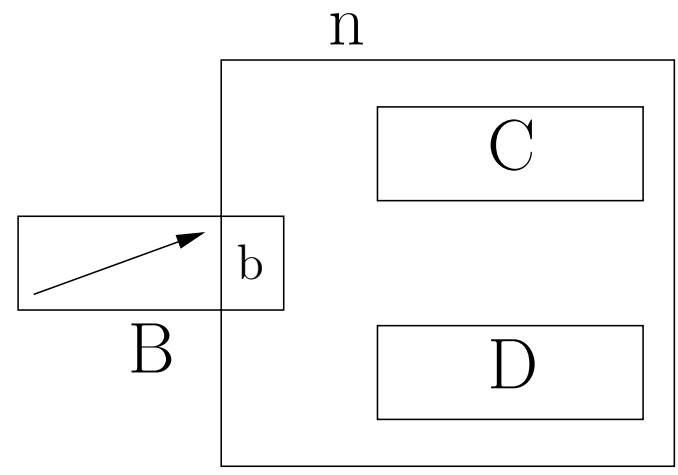

Figure 4b: $\tilde{b}, C$ and $D$ avoids 1342 and 2143

$\mathrm{n}$
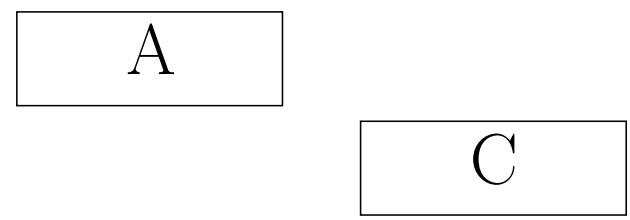

Figure 4c: $A$ avoids 1342 and 2143 and $C$ avoids 1342 and 2143

smaller block on the right, we again see that $B$ must be an increasing block. Moreover, if we let the largest number in $B$ be $\tilde{b}$, then for the permutation to avoid 1342 and 2143, it will be necessary and sufficient for the subsequence formed by $\tilde{b}, C$, and $D$ to avoid 1342 and 2143 [Figure 4b].

For this case, we may count the number of permutations as follows. Let $i$ be the length of $B$. Then because the subsequence formed by $\tilde{b}, C, D$ can be any element of $S_{n-i}(1342,2143)$ that doesn't begin with $n$. There are $c_{n-i}-c_{n-i-1}$ of these. Summing from $i=1$ to $n-2$, this telescopes to $c_{n-1}-1$. Thus the number of permutations of length n avoiding 1342 and 2143 and containing one block on the left of $n$ and a larger one to the right is $c_{0}\left(c_{n-1}-1\right)$ (by convention we take $c_{0}$ to be 0 ).

Case 3: The numbers to the left of $n$ are all greater than all those to the right of $n$ (this hypothesis is vacuous if there are no elements to the left of $n$ or no elements on the right). Note that this covers the cases with one block on the left that were excluded in Case 2 and the cases with no blocks on the left.

In this case, $n$ cannot act as a 4 for either 1342 or 2143 , because in neither permutation are the elements on the right of 4 all bigger than the elements on the left. Moreover, because there is no way of breaking up either 1342 or 2143 into two non-empty halves with the first half greater than the second, any 1342 or 2143 subsequence must occur either entirely to the left or entirely to the right of $n$.

If there are $i$ elements to the left of $n$ and $n-i-1$ elements to the right, then summing over $i$ gives $\sum_{i=0}^{n-1} c_{i} c_{n-i-1}$ possibilities for this case [Figure 4c]. Note that if we call the 

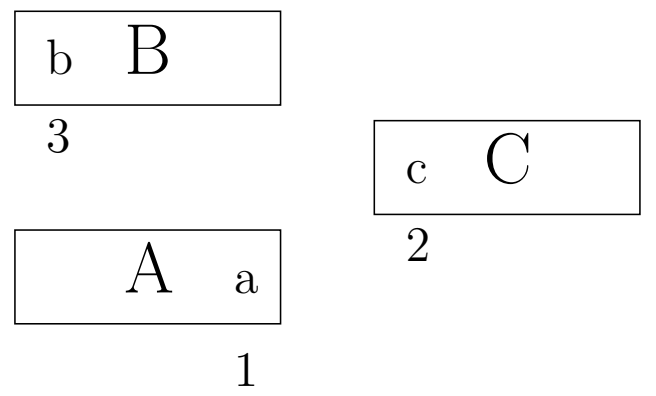

Figure 5: banc is an occurrence of 3142

left (possibly empty) block $A$ and the right (possibly empty) block $C$, we can consider this similar to Case 1 except with block $B$ empty (and block $A$ possibly empty as well).

Finally, adding together these three cases we get the recurrence

$$
c_{n}=\sum_{i=0}^{n-1} c_{i}\left(2 c_{n-i-1}-1\right)
$$

for $n \geq 1$ (we take by convention $c_{0}=1$ ). Translating this into generating function notation, we find that if $f(x)=\sum c_{n} x^{n}$, then

$$
x f\left(2 f-\frac{1}{1-x}\right)=f-1 .
$$

This is quadratic equation in $f$, and solving gives

$$
f=\frac{1-\sqrt{1-8 x+16 x^{2}-8 x^{3}}}{4 x(1-x)}
$$

where we take the minus sign so that $f(0)=1$.

\subsection{Permutations in $S_{n}(3142,2341)$}

We can proceed similarly to count the number of permutations in $S_{n}(3142,2341)$. The steps are entirely analogous to the case of $S_{n}(1342,2143)$, and the bijection between the sets $S_{n}(3142,2341)$ and $S_{n}(1342,2143)$ will be clear from the proof.

Any permutation $\sigma \in S_{n}(3142,2341)$ has two or fewer blocks to the left of $n$. First consider any two distinct blocks $A$ and $B$ to the left of $n$. Without loss of generality, $A<B$. Then because $A$ and $B$ are distinct blocks, there is a block $C$ to the right of $n$ such that $B>C>A$. Now if any element $b \in B$ occurred before an element $a \in A$ [Figure 5], then we would have the subsequence banc contained in $\sigma$. But $a<c<b<n$, so that banc would be an occurrence of 3142 . Thus all numbers in the block $B$ lie to the 


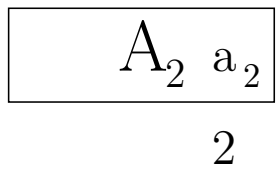

2

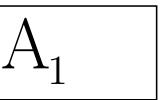

2

(1)

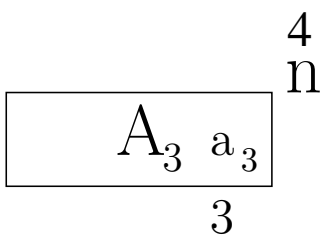

b B

1

Figure 6: $a_{2} a_{3} n b$ is an occurrence of 2341

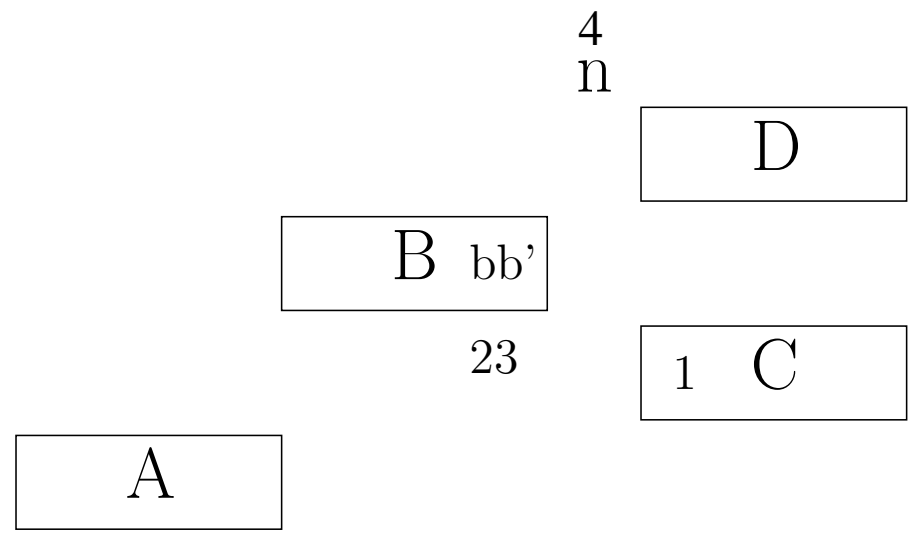

Figure 7: $b b^{\prime} n c$ is an occurrence of 2341

right of all the numbers in block $A$. Since $A$ and $B$ could be any blocks to the left of $n$, all the blocks on the left occur in increasing order.

Now suppose there were three or more blocks on the left hand side. Let $A_{1}, A_{2}, A_{3}$ be three of these blocks, with $A_{1}<A_{2}<A_{3}$ so that they occur in the order $A_{1}, A_{2}, A_{3}$ [Figure 6]. Then because $A_{1}$ and $A_{2}$ are distinct, there is some block $B$ to the right of $n$ between them, i.e., $A_{1}<B<A_{2}$. Then if we have $a_{2} \in A_{2}, a_{3} \in A_{3}$ and $b \in B$, then $b<a_{2}<a_{3}<n$, so that the subsequence $a_{2} a_{3} n b$ would be an occurrence of 2341 . Thus there are two or fewer blocks on the left side of $n$, and they are in increasing order.

We now proceed to count the number of permutations $\sigma \in S_{n}(3142,2341)$. We break this up into three cases.

Case 1: There are two blocks $A<B$ to the left of $n$.

First we show there is no element $x$ on the right-hand side of $n$ such that $x<A$. For if there were, taking $a \in A$ and $b \in B$, we would have that abnx is an occurrence of 2341 . 


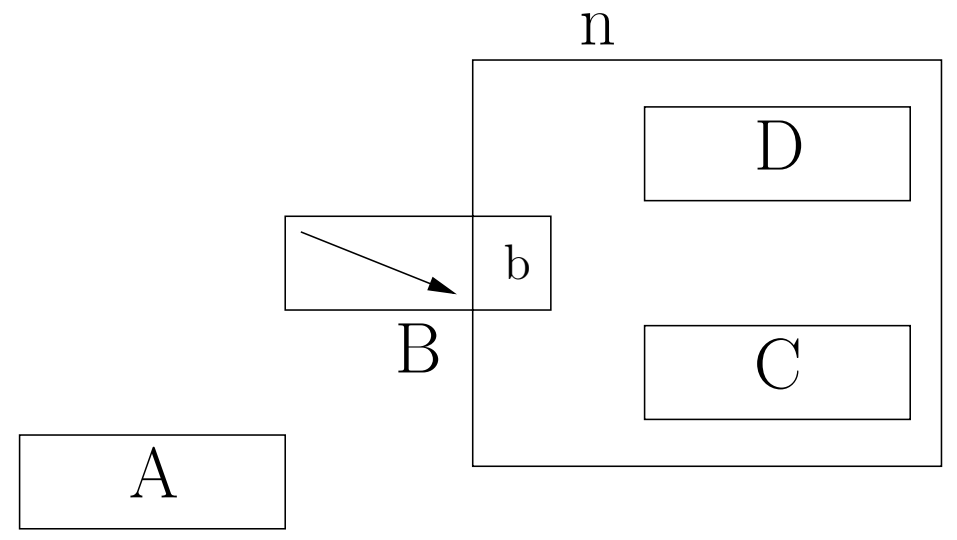

Figure 8a: $A$ avoids 3142 and 2341 and $\tilde{b}, C$ and $D$ avoids 3142 and 2341

Thus on the right side of $n$ there are at most two blocks: there must be a block $C$ with $B>C>A$ and there is possibly a block $D$ with $D>B$. Moreover, the numbers in the block $B$ must occur in decreasing order, for if $B$ contained an increasing subsequence $b b^{\prime}$, then, taking $c$ in $C$, we would have $b b^{\prime} n c$ an occurrence of 2341 [Figure 7]. Let $\tilde{b} \in B$ be the the smallest element of $B$. Now clearly the block $A$ must avoid 3142 and 2341, and the subsequence of $\sigma$ formed by $\tilde{b}, C$ and $D$ must avoid 3142 and 2341 . We claim that this is a sufficient condition for $\sigma$ to avoid 3142 and 2341 [Figure 8a].

Proposition 4 In the notation above, if the elements of $B$ occur in decreasing order, $A$ avoids 3142 and 2341 and the subsequence of $\sigma$ formed by $\tilde{b}, C$ and $D$ avoids 3142 and 2341, then the permutation $\sigma$ avoids 3142 and 2341.

Proof of Proposition 4: The proof of this follows as the proof of Proposition 3 above, with 1342 replaced by 3142, 2143 replaced by 2341, "increasing" and "decreasing" interchanged, 3 and 1 interchanged and $<$ and $>$ interchanged.

We will find as before that if $d_{n}=\left|S_{n}(3142,2341)\right|$, then the number of permutations in this case is

$$
\sum_{i=1}^{n-1} d_{i}\left(d_{n-i-1}-1\right) .
$$

Case 2: There is one block to the left of $n$, and there is a smaller block to the right of $n$

As in Case 2 for the the permutations 1342 and 2143, this reduces to Case 1 with the length of block $A$ equal to zero. Letting $B$ be the block on the left of $n, C$ be the smaller block on the right, and $D$ the possibly empty larger block on the right, we see that $B$ must be an decreasing block. Moreover, if we let the smallest number in $B$ be $\tilde{b}$, then for the permutation to avoid 3142 and 2341, it will be necessary and sufficient for the subsequence formed by $\tilde{b}, C$, and $D$ to avoid 3142 and 2341 [Figure 8b]. Then because the subsequence formed by $\tilde{b}, C$ and $D$ can be any element of $S_{n-i}(1342,2143)$ that doesn't begin with 1. 


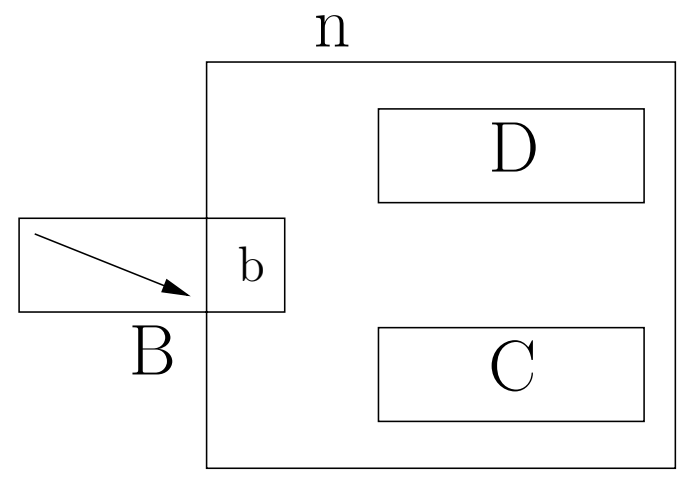

Figure 8b: $\tilde{b}, C$ and $D$ avoids 3142 and 2341

n

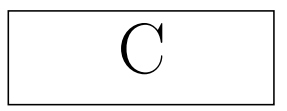

\section{A}

Figure 8c: $A$ avoids 3142 and 2341 and $C$ avoids 3142 and 2341

There are $d_{n-i}-d_{n-i-1}$ such permutations in $S_{n-1}$. Summing from $i=1$ to $n-2$, this telescopes to $d_{n-1}-1$. Thus the number of permutations of length $n$ avoiding 3142 and 2341 and containing one block on the left of $n$ and a larger one to the right is $d_{0}\left(d_{n-i}-1\right)$ (here we take $d_{0}=0$ by convention).

Case 3: The numbers to the left of $n$ are all smaller than all those to the right of $n$ (this hypothesis is vacuous if there are no elements to the left of $n$ or no elements on the right). Note that this covers the cases with one block on the left that were excluded in Case 2 and the cases with no blocks on the left.

In this case, $n$ cannot act as a 4 for either 3142 or 2341 , because in neither permutation are the elements on the right of 4 all smaller than the ones on the left. Moreover, because there is no way of breaking up either 3142 or 2341 into two non-empty halves with the first half smaller than the second, any 3142 or 2341 subsequence must occur either entirely to the left or entirely to the right of $n$ [Figure 8c].

This gives $\sum_{i=0}^{n-1} d_{i} d_{n-i-1}$ possibilities for this case. Note that if we call the left (possibly empty) block $A$ and the right (possibly empty) block $C$, we can consider this similar to Case 1 except with block $B$ empty (and block $A$ possibly empty as well).

So, we find that $\left|S_{n}(1342,2143)\right|$ and $\left|S_{n}(3142,2341)\right|$ satisfy the same recurrence, and because $\left|S_{0}(1342,2143)\right|=\left|S_{0}(3142,2341)\right|=1$, we have $c_{n}=\left|S_{n}(1342,2143)\right|=$ $\left|S_{n}(3142,2341)\right|$ for all $n$, and moreover

$$
\sum c_{n} x^{n}=\frac{1-\sqrt{1-8 x+16 x^{2}-8 x^{3}}}{4 x(1-x)} .
$$


Inductively, we can construct a bijection between $S_{n}(1342,2143)$ and $S_{n}(3142,2341)$, that maps permutations in $S_{n}(1342,2143)$ that do not begin with $n$ to permutations in $S_{n}(3142,2341)$ that do not begin with 1 . We have the trivial correspondence between $S_{1}(1342,2143)$ and $S_{1}(3142,2341)$. Now suppose that for $k<n$, we have a bijection between $S_{k}(1342,2143)$ and $S_{k}(3142,2341)$ that maps permutations in in $S_{k}(1342,2143)$ that do not begin with $k$ to permutations in $S_{k}(3142,2341)$ that do not begin with 1 . Then we may construct a bijection between $S_{n}(1342,2143)$ and $S_{n}(3142,2341)$ by using the bijection for $k<n$ for the subsequence $A$ and the subsequence $\tilde{b}, C$ and $D$ in Cases 1 and 2. In Case 3, we do the same for the blocks $A$ and $C$. Then we end up with a bijection that takes permutations in $S_{n}(1342,2143)$ that do not begin with $n$ to permutations in $S_{n}(3142,2341)$ that do not begin with $n$ (and takes permutations beginning with $n$ to permutations beginning with $n)$. But the permutations in $S_{n}(3142,2341)$ that begin with 1 and the permutations in $S_{n}(3142,2341)$ that begin with $n$ can be interchanged in a canonical way: if $\sigma \in S_{n}(3142,2341)$ begins with $n$, we can change the $n$ to a 1 and add one to the remaining numbers in the permutation to get a permutation in $S_{n}(3142,2341)$ that begins with 1 . Moreover the process is reversible. This gives a bijective map from $S_{n}(1342,2143)$ to $S_{n}(3142,2341)$, that takes permutations that do not begin with $n$ to permutations that do not begin with 1 .

We now move on to the next theorem.

Proof of Theorem 2: Let $S_{n k}(1342,3124)$ denote the set of permutations of length $n$ that begin with a descending sequence of length $k$, but not a descending sequence of length $k+1$. Similarly defining $S_{n k}(1243,2134)$, we will show that

$$
\left|S_{n k}(1342,3124)\right|=\left|S_{n k}(1243,2134)\right| .
$$

\subsection{The Structure of permutations in $S_{n}(1342,3124)$}

To begin the proof, consider a permutation $\sigma \in S_{n}(1342,3124)$. Note that in the argument above for the case of $S_{n}(1342,2143)$, we used only the fact that a permutation avoided 1342 to conclude that the blocks to the left of $n$ are in decreasing order. Let $A_{1}, A_{2}, \ldots, A_{s}$ be these blocks in decreasing order. Note that for $i>1$, the block $A_{i}$ must be strictly decreasing, because if $b, c \in A_{i}, b<c$ occur in increasing order, then taking $a \in A_{i-1}$ we would have that abcn is a 3124 subsequence [Figure 9]. We now consider two cases.

Case 1: $s>1$.

Let $B_{i}, 1 \leq i \leq s$ be the blocks on the right of $n$ that separate the $A_{i}$, i.e. $A_{i}<$ $B_{i}<A_{i-1}$ ( $B_{1}$ may be empty). There may be an additional block $C$ to the right of $n$ consisting of numbers smaller than $A_{s}$. Also, let $a \in A_{s}$ be the smallest number to the left of $n$, i.e., the last element of $A_{s}$. The $B_{i}$ must occur in decreasing order. For if we had $b_{i+1} \in B_{i+1}$ occurring before $b_{i} \in B_{i}$, then taking $a_{i} \in A_{i}$ we would have the 3124 subsequence $a_{i} a b_{i+1} b_{i}$ [Figure 10]. Moreover, for $i<s$, the block $B_{i}$ must be strictly decreasing, for the same reason that for $i>1$, the block $A_{i}$ must be strictly decreasing, 

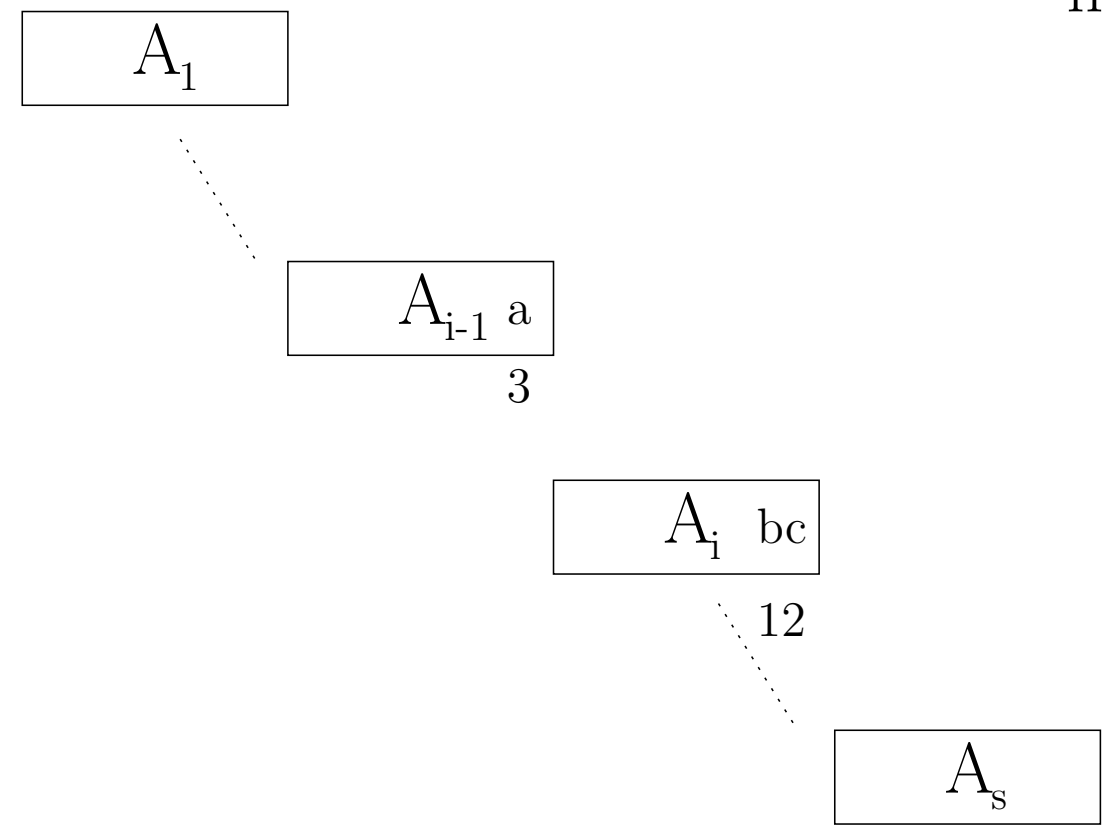

Figure 9: abcn is a 3124 subsequence

namely if $b, c \in B_{i}, i<s$ occur in increasing order, then taking $d \in B_{i+1}$, we get that abcd is a 1342 subsequence [Figure 11].

The block $A_{1}$ must avoid 1342 and 312, because adding $n$ to any 312 sequence in $A_{1}$ creates a 3124 sequence. Moreover, it is clear that we must have the subsequence consisting of $a, B_{s}$ and $C$ avoid 1342 and 3124. Let us denote this subsequence by $D$. Now, the blocks $B_{i}, i<s$ may be interspersed with the block $D$. However, this may not be done arbitrarily. Let $d_{1} d_{2}$ be the first (in the sense that $d_{2}$ occurs as early as possible) occurring increasing subsequence of length two in $D$. This means that up until $d_{2}$, the subsequence $D$ is strictly decreasing. We claim that the blocks $B_{i}, i<s$ must occur before $d_{2}$. Because $a<B_{s}, d_{2}$ is either the first element of $B_{s}$ or it is in $C$. Suppose $d_{2}$ is the first element of $B_{s}$. Clearly $B_{i}, i<s$, must occur before $B_{s}$ by the argument in the preceding paragraph. Suppose $d_{1} \in C$. Then if $b \in B_{i}, i<s$ occurred after $d_{2}$, then $a d_{1} d_{2} b$ would be a 3124 subsequence [Figure 12a].

To summarize, we have that for any permutation $\sigma \in S_{n}(1342,3124)$, the following conditions hold under the conditions of Case 1:

1. the blocks $A_{i}$ and $B_{i}$ are arranged in decreasing order

2. the blocks $A_{i}, i>1$ and $B_{i}, i<s$ are themselves decreasing

3. the block $A_{1}$ avoids 1342 and 312 
n

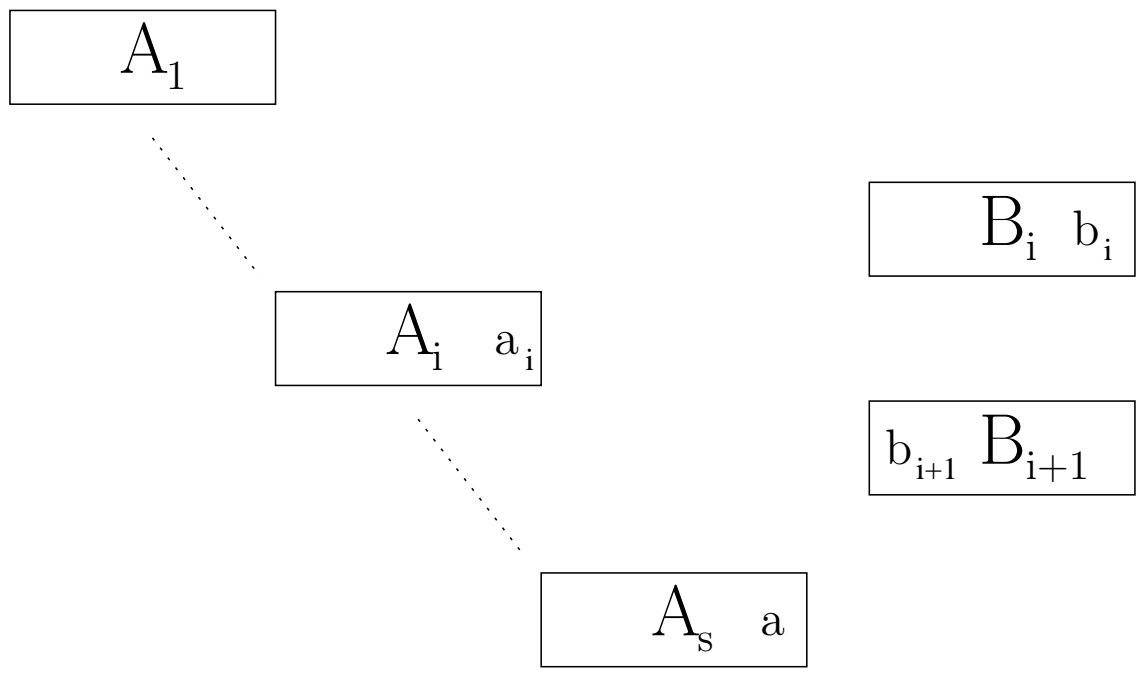

Figure 10: $a_{i} a b_{i+1} b_{i}$ is a 3124 subsequence

n

$\mathrm{B}_{\mathrm{i}} \mathrm{bc}$

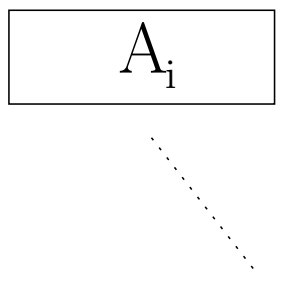

$$
\text { d } \mathrm{B}_{\mathrm{i}+1}
$$

$A_{\mathrm{s}}$ a

Figure 11: abcd is a 1342 subsequence 


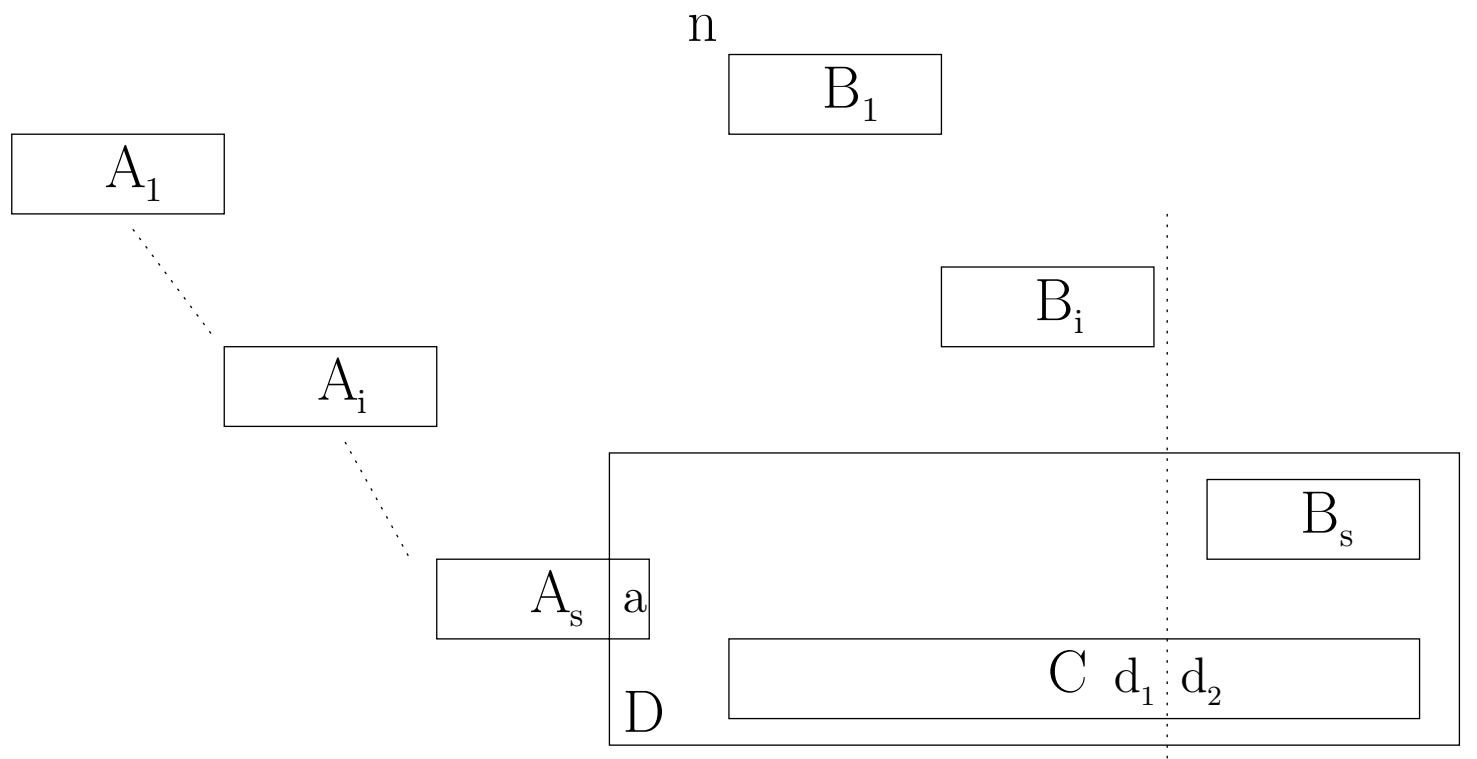

Figure 12a: $A_{1}$ avoids 1342 and 312 and $D$ avoids 1342 and 3124

4. the subsequence $D$ (consisting of $a, B_{s}$ and $C$ ) avoids 1342 and 3124, and $a$, the first element of $D$, is not the largest element of $D$

5. the blocks $B_{i}, i<s$ are interspersed so that they occur to the right of $n$ and to the left of $d_{2}$, the last number in $D$ such that all the elements of $D$ to the left of $d_{2}$ are arranged in decreasing order

Note that only the blocks $B_{1}$ and $C$ may be empty-all others must contain elements. In the proof we will assume implicitly the structure given by conditions 1,2 and 5 and use conditions 3,4 to show that any $\sigma$ satisfying conditions 1 through 5 avoids 1342 and 3124 .

Case 2: $s=1$ and there are elements to the right of $n$ greater than elements on the left of $n$.

If there is one block $A_{1}$ to the left of $n$, it must avoid 1342 and 312 . If we call the smallest element of this block $a$, then there are possibly two blocks $B$ and $C$ to the right of $n$. We may chose them such that $C<A_{1}<B$. The subsequence $D$ formed by $a, B$ and $C$ must avoid 1342 and 3124. This is sufficient. In fact, these are the same conditions as in the case $s>1$ if we take $B_{1}=B$. Conditions 1,2 and 5 are vacuously fulfilled. Note that and $C$ may be empty [Figure 12b].

In the case that $B$ is empty, we have

Case 3: $s=1$ and all the elements to the left of $n$ (the elements of $A_{1}$ ) are greater than those to the right of $n$.

In this case $A_{1}$ must avoid 1342 and 312. There is possibly a block $C$ to the right of $n$. It must avoid 1342 and 3124. This is sufficient, using an argument along the lines of the one above for Case 2 [Figure 12c]. 


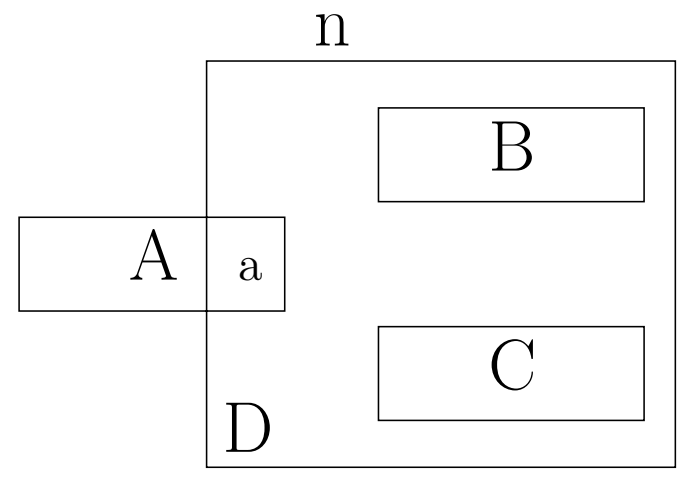

Figure 12b: $A_{1}$ avoids 1342 and 312 and $D$ avoids 1342 and 3124

$\mathrm{n}$
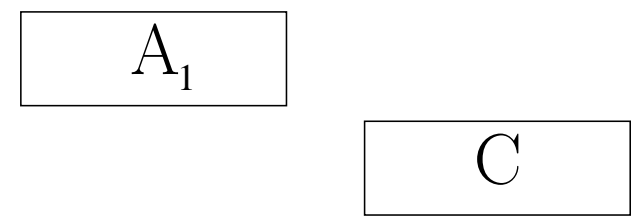

Figure 12c: $A_{1}$ avoids 1342 and 312 and $C$ avoids 1342 and 3124

Proposition 5 In the notation above, for $s \geq 1$, conditions 1 through 5 suffice to guarantee that $\sigma$ avoids 1342 and 3124.

We also have the following simple case to take care of before we proceed with the proof of Proposition 5.

Case 4: $s=0$.

If $s=0$ then the permutation consists of $n$ appended to the left of any permutation in $S_{n-1}(1342,3124)$. It is clear that all such permutations avoid 1342 and 3124 and that any permutation with no blocks on the left must have this form.

Proof of Proposition 5: We proceed by case analysis. Let $\sigma$ satisfy conditions 1 through 5 Then suppose that $\sigma$ contains a 3124 subsequence. If the 4 were $n$, then there would be a 312 subsequence among the $A_{i}$. However, as the elements in $A_{i}, i>1$ are all arranged in decreasing order, the 2 must occur within $A_{1}$, and hence the 312 must occur in $A_{1}$, contradicting condition 3 Hence the 4 in a 3124 subsequence is not $n$. Moreover, the above reasoning shows that the there is no 312 subsequence to the left of $n$, so that any 4 must occur to the right of $n$.

Suppose that the 4 occurs within the $B_{i}, i<s$. Then because the elements of $B_{i}, i<s$ are arranged in decreasing order, the 3,1 , and 2 all occur in $C$ or the $A_{i}$. If the 1 is in the $A_{i}$, then so is the 3 , but then the 2 must lie in among the $B_{i}, i<s$, which is impossible, because the 2 cannot occur before the 4 , as the $B_{i}$ are strictly decreasing. So either the 3 occurs in the $A_{i}$ and the 12 occurs in the $C$ or the 312 occurs in $C$. However, in each 
of these cases, we may take the 4 in $B_{s}$ and the 3 to be $a$. But condition 4 tells us that the the subsequence $D$ formed by $a, B_{s}$, and $C$ avoids 3124 , so we have a contradiction, and the 4 cannot be among the $B_{i}, i<s$.

If the 4 occurs in $B_{s}$, then the 312 occurs among the $A_{s}$ and $C$. The 312 cannot occur in $A_{s}$, as noted above, and if both the 3 and the 1 occurred in $A_{s}$, the 2 would have to as well. Thus, at most one element of $A_{s}$ is used, we may as well take $a$. But the subsequence $D$ formed by $a, B_{s}$ and $C$ avoids 3124 by 4 . Finally, if the 4 occurs in $C$, then the whole subsequence lies in $C$, again contradicting condition 4 .

Now suppose that there were a 1342 subsequence in $\sigma$. If the 3 were to the left of $n$, the 1 would be also. But the only possible increasing subsequences lie in $A_{1}$, so that both the 1 and 3 would lie there, forcing the 2 and thus also the 4 to lie there, contradicting condition 3 , the statement that $A_{1}$ avoids 1342 . The 3 and 4 form an increasing sequence, so they must lie in $B_{s}$ or $C$. In either case, that would force the 1 to lie in $A_{1}$ or $C$. In the former case we could let the 1 be $a$. Either way, we end up with a 1342 sequence in $D$, a contradiction.

Thus we have an understanding of the structure of all permutations in $S_{n}(1342,3124)$. We shall see that the structure of permutations in $S_{n}(1243,2134)$ is very similar.

\subsection{The Structure of Permutations in $S_{n}(1243,2134)$}

Let $\sigma \in S_{n}(1243,2134)$. Let $x$ denote the greatest number to the right of $n$. We consider four cases analogous to the four cases in the previous section.

Case 1: There are two or more numbers to the left of $n$ that are less than $x$.

All the numbers to the left of $n$ that are less than $x$ must occur in decreasing order. For if $a, b$ occur in increasing order and to the left of $n$, then $a b n x$ is a 1243 subsequence. From this point on, let $a$ denote the smallest element to the left of $n$. Then the other elements to the left of $n$ are arranged in blocks $\tilde{A}_{1}, \tilde{A}_{2}, \ldots, \tilde{A}_{s-1}$, with $\tilde{A}_{1}>\tilde{A}_{2}>\cdots>\tilde{A}_{s-1}$. Although a may or may not be in the same block as $\tilde{A}_{s-1}$, for now we will decree that $a \notin \tilde{A}_{s-1}$, so that $\tilde{A}_{s-1}$ may not actually be a block as defined before. There also may be some block $\tilde{A}_{0}$ to the left of $n$ that is greater than $x$. However, no element of this block can occur after the second element of the $A_{i}, i \geq 1$. For if $a_{0} \in \tilde{A}_{0}$ occurred after $a^{\prime} a^{\prime \prime}$ in $\tilde{A}_{i}, i \geq 1$, then $a^{\prime} a^{\prime \prime} a_{0} n$ would be a 2134 subsequence [Figure 13].

On the right of $n$, there are blocks $x \in \tilde{B}_{1}, \tilde{B}_{2}, \ldots, \tilde{B}_{s}, C$, with $\tilde{A}_{0}>\tilde{B}_{1}>\tilde{A}_{1}>\tilde{B}_{2}>$ $\cdots>\tilde{A}_{s-1}>\tilde{B}_{s}>a>C$. Now $\tilde{B}_{s}$ may or may not be empty depending on whether $a$ is part of the block $\tilde{A}_{s-1}$. Also, elements in the blocks $\tilde{B}_{i}, i<s$, are arranged in decreasing order. For if $b b^{\prime}$ is an increasing sequence in the $\tilde{B}_{i}, i<s$, then taking $a_{s-1} \in \tilde{A}_{s-1}$ we would get that $a_{s-1} a b b^{\prime}$ is a 2134 subsequence [Figure 14]. Now, by analogy with the block $\tilde{A}_{0}$, no elements of $\tilde{B}_{s}$ may occur before the second to last element among the $\tilde{B}_{i}, i<s$. For if $b_{s} \in \tilde{B}_{s}$ occurs before $b b^{\prime} \in \tilde{B}_{i}, i<s$, then $a b_{s} b b^{\prime}$ is a 1243 subsequence [Figure 15].

To summarize, we have concluded that in $\sigma$, we have blocks $\tilde{A}_{0}>\tilde{A}_{1}>\cdots>\tilde{A}_{s-1}$ on the left of $n$ and blocks $\tilde{B}_{1}>\cdots>\tilde{B}_{s}$ on the right of $n$. Every element of $\tilde{A}_{0}$ occurs before the second element of $A_{1}$. Similarly, every element of $\tilde{B}_{s}$ occurs after the second-to-last element of $\tilde{B}_{s-1}$. Moreover, the $\tilde{A}_{i}, i>0$, occur in decreasing order, and the $\tilde{B}_{i}, i<s$, 


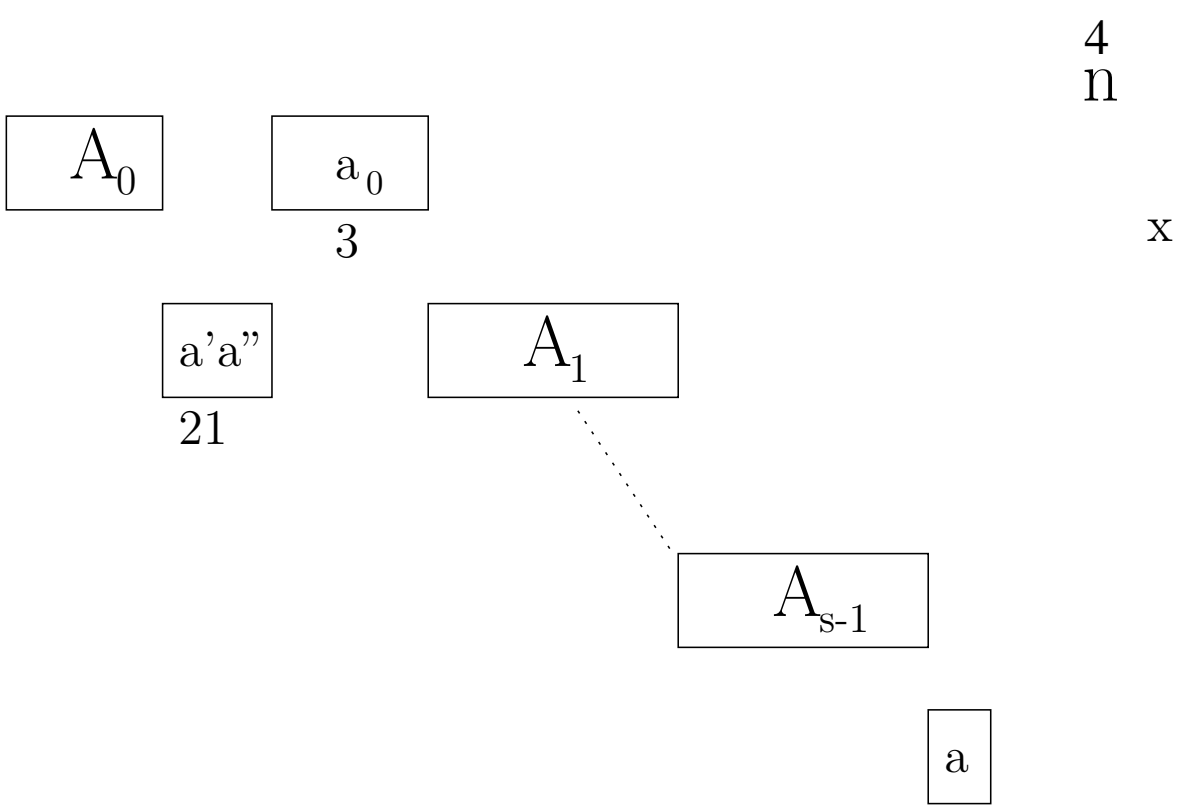

Figure 13: $a^{\prime} a^{\prime \prime} a_{0} n$ is a 2134 subsequence

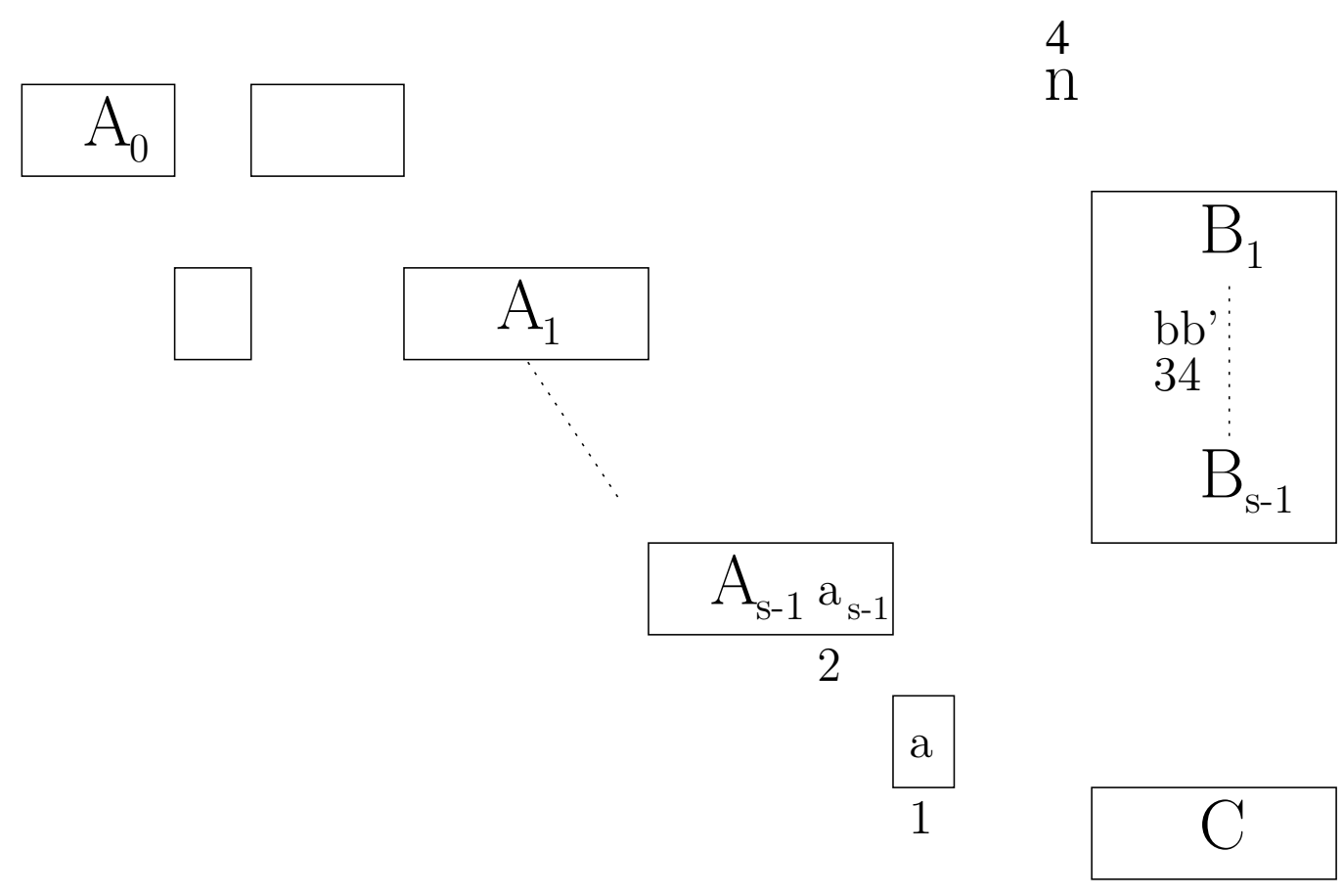

Figure 14: $a_{s-1} a b b^{\prime}$ is a 2134 subsequence 


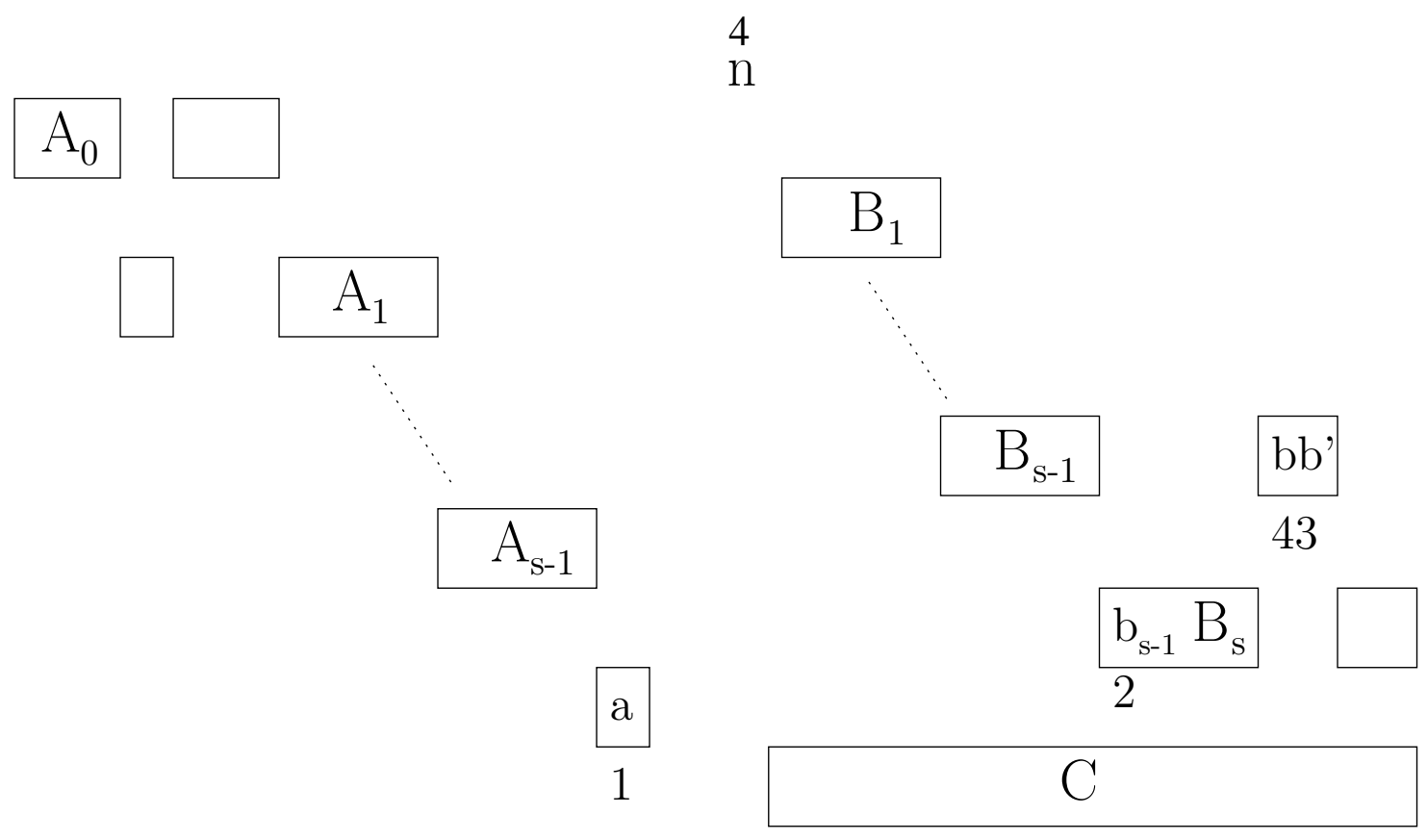

Figure 15: $a b_{s} b b^{\prime}$ is a 1243 subsequence

also occur in decreasing order. Furthermore $a$, which may or may not be a part of $\tilde{A}_{s-1}$ occurs directly before $n$. Finally, there may be a block $C$ smaller than $a$, so that we have the order relations $n>\tilde{A}_{0}>\tilde{B}_{1}>\tilde{A}_{1}>\tilde{B}_{2}>\cdots>\tilde{A}_{s-1}>\tilde{B}_{s}>a>C$. Only $B_{s}, A_{0}$, and $C$ may be empty-all other blocks must contain elements.

Henceforth, let $a_{i} \in \tilde{A}_{i}$ be the largest element of $A_{i}$ for $i>0$, and $b_{i} \in \tilde{B}_{i}$ be the smallest element of $\tilde{B}_{i}$ for $i<s$. Then let $A_{1}=\tilde{A}_{0} \cup\left\{a_{1}\right\}, A_{i}=\tilde{A}_{i-1} \backslash\left\{a_{i-1}\right\} \cup\left\{a_{i}\right\}$, $0<i<s$, and $A_{s}=\tilde{A}_{s-1} \backslash\left\{a_{s-1}\right\} \cup\{a\}$. Also let $B_{1}=\tilde{B}_{1} \backslash\left\{b_{1}\right\}, B_{i}=\tilde{B}_{i} \backslash\left\{b_{i}\right\} \cup\left\{b_{i-1}\right\}$, and $B_{s}=\tilde{B}_{s} \cup\left\{b_{s-1}\right\}$. From the previous paragraph, in $\sigma$ these sets occur in the order $A_{1}, A_{2}, \ldots, A_{s}$ and $B_{1}, B_{2}, \ldots, B_{s}$, analogous to the situation in $S_{n}(1342,3124)$. Moreover, only $B_{1}$ and $C$ may be empty.

Now it is clear that the set $A_{1}$ must avoid 1243 and 213 because any 213 sequence, after adding $n$, gives a 2134 subsequence. Additionally, the subsequence formed by $a, B_{s}$, and $C$, call it $D$, must avoid 1243 and 2134. Now, the sets $B_{i}, i<s$, may be interspersed with the block $D$. However, this may not be done arbitrarily. Let $d_{1} d_{2}$ be the first (in the sense that $d_{2}$ occurs as early as possible) occurring increasing subsequence of length two in $D$. This means that up until $d_{2}$, the subsequence $D$ is strictly decreasing. We claim that the blocks $B_{i}, i<s$ must occur before $d_{2}$. We know that $d_{2}$ is either in $C$ or it is the first element of $B_{s}$, because $a<B_{s}$. Suppose $d_{2}$ is the first element of $B_{s}$. Then, as noted above, $B_{i}, i<s$, must occur before $B_{s}$, or before $d_{2}$.

Now suppose $d_{2} \in C$. Then if $b \in B_{i}, i<s$ occurred after $d_{2}$, then $d_{1} d_{2} b b_{s-1}$ would be a 1243 subsequence. Thus, the sets $B_{i}, i<s$ are interspersed within the subsequence $D$ before $d_{2}$ [Figure 16a]. 


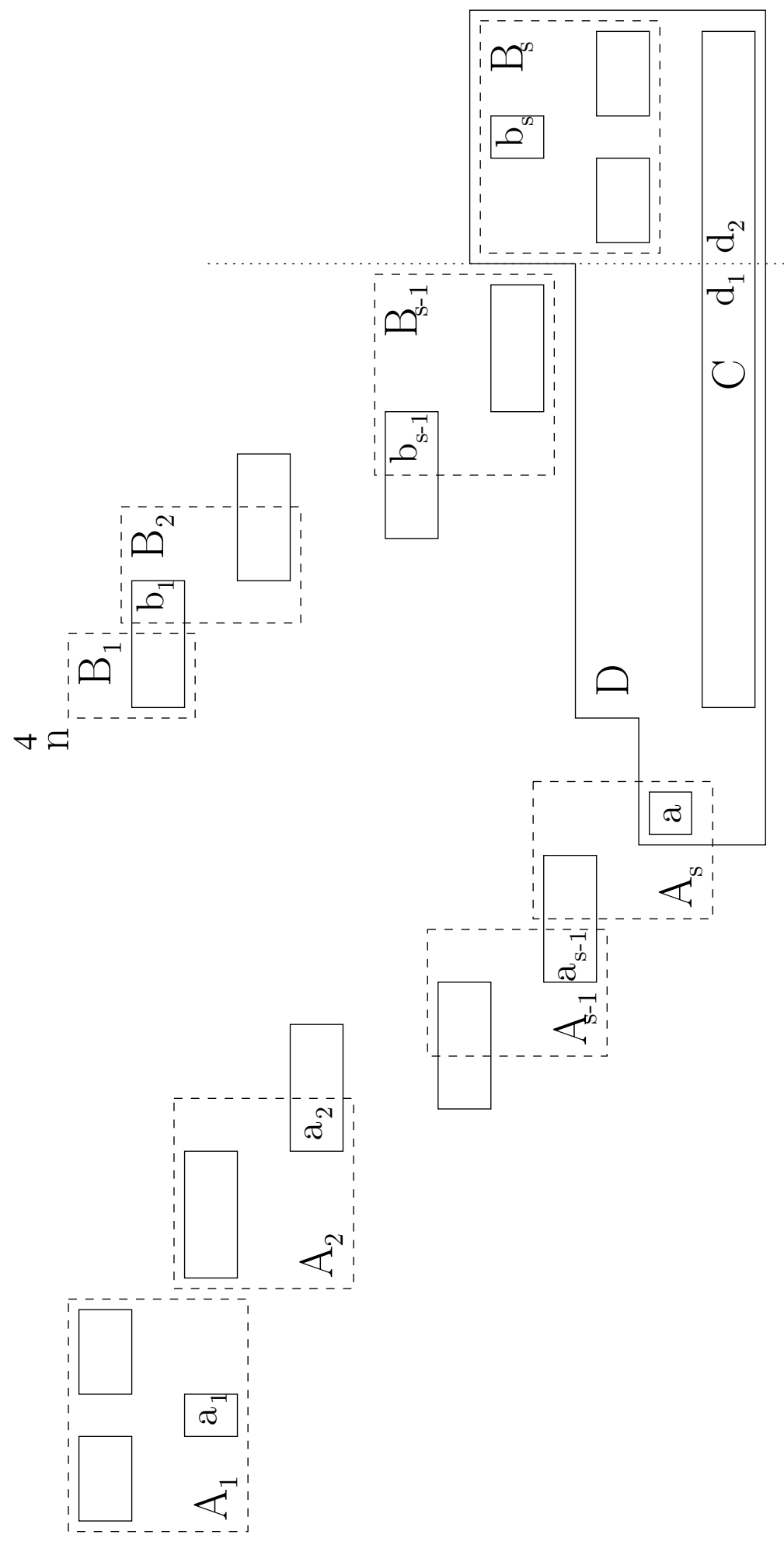

Figure 16a: $A_{1}$ avoids 1243 and 213 and $D$ avoids 1243 and 2134 


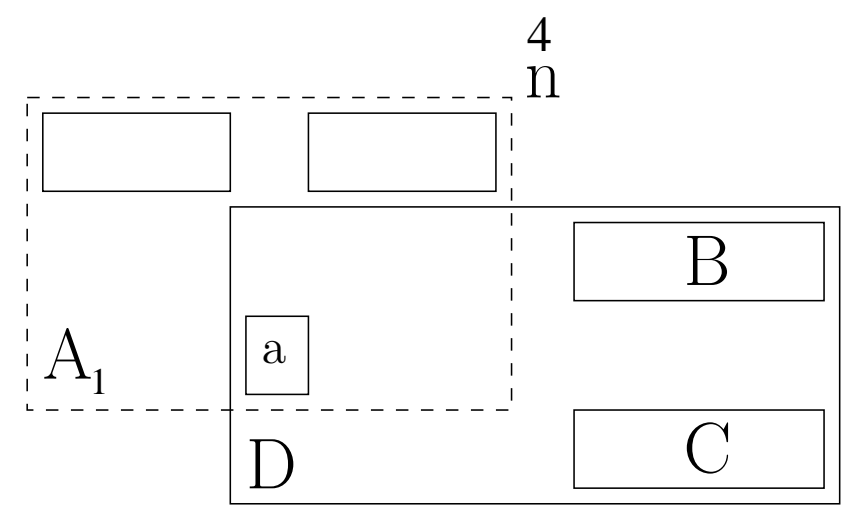

Figure 16b: $A_{1}$ avoids 1243 and 213 and $D$ avoids 1243 and 2134

To summarize, we have that for any permutation $\sigma \in S_{n}(1243,2134)$, the sets $A_{i}, B_{i}$ defined as above from the blocks $\tilde{A}_{i}$ and $\tilde{B}_{i}$ satisfy the following conditions:

1. the $A_{i}$ and $B_{i}$ are arranged in decreasing order

2. the sets $A_{i}, i>1$ and $B_{i}, i<s$ are themselves decreasing

3. the block $A_{1}$ avoids 1342 and 312

4. the subsequence $D$ (consisting of $a, B_{s}$, and $C$ ) avoids 1243 and 2134, and $a$, the first element of $D$, is not the largest element of $D$

5. the $B_{i}, i<s$ are interspersed so that they occur to the right of $n$ and to the left of $d_{2}$, the last number in $D$ such that all the elements of $D$ to the left of $d_{2}$ are arranged in decreasing order

Note that only the sets $B_{1}$ and $C$ may be empty-all others must contain elements. In the proof we will assume implicitly the structure given by conditions 1,2 and 5 and use conditions 3 and 4 to show that any $\sigma$ satisfying conditions 1 through 5 avoids 1243 and 2134. Note that this is almost completely analogous to the case $S_{n}(1342,3124)$, except that

- here $D$ avoids 1243 and 2134 instead of 1342 and 3124

- $A_{1}$ avoids 1243 and 213 instead of 1342 and 312

- the $A_{i}$ and $B_{i}$ have different vertical relationship-whereas before $B_{i}>A_{i}>B_{i+1}$, here we have that $b_{i-1}>a_{i-1}>A_{i} \backslash\left\{a_{i}\right\}>B_{i} \backslash\left\{b_{i-1}\right\}>b_{i}>a_{i}$.

Case 2: There is exactly one number to the left of $n$ less than $x$.

Let this number be $a$. There may be some numbers to the left of $n$ greater than $x$. Now all the numbers to the left of $n$ must avoid 1243 and 213 . Let $B$ and $C$ be the two 
$\mathrm{n}$
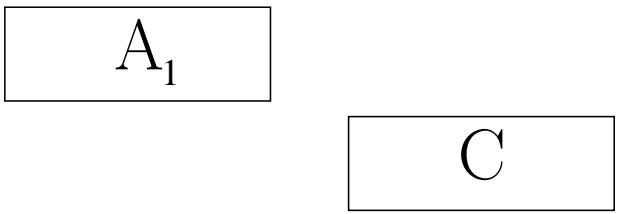

Figure 16c: $A_{1}$ avoids 1243 and 213 and $C$ avoids 1243 and 2134

blocks to the right of $n$, with $x \in B>a>C$. The subsequence $D$ formed by $a$, $B$, and $C$ must avoid 1243 and 2134. This turns out to be sufficient. In fact, these are the same conditions as in the case with more than one number to the left of $n$ less than $x$, if we take all the numbers to the left of $n$ to be $A_{1}$, and $B_{1}=B$. Conditions 1,2 and 5 are vacuously fulfilled. Note that $C$ may be empty [Figure 16b]. Corresponding to the case where $B$ is empty in the $S_{n}(1342,3124)$ case is

Case 3: There are no numbers to the left of $n$ less than $x$, only numbers larger than $x$.

Here, we need that the numbers before $n$ avoid 1243 and 213 and the numbers to the right to avoid 1243 and 2134. This is analogous to Case 3 for $S_{n}(1342,3124)$, where we needed the numbers to the left to avoid 1342 and 312 and the numbers to the right to avoid 1342 and 312 [Figure 16c]. Finally there is

Case 4: There are no numbers to the left of $n$.

In this case the permutation consists of $n$ appended to the left of any permutation in $S_{n-1}(1243,2134)$. It is clear that all such permutations avoid 1243 and 2134 and that any permutation with no blocks on the left must have this form.

Proposition 6 In the notation above, for Cases 1 and 2, conditions 1 through 5 suffice to guarantee that $\sigma$ avoids 1243 and 2134.

Proof of Proposition 6: We proceed by case analysis. Let $\sigma$ satisfy conditions 1 through 5. Then suppose that $\sigma$ contains a 2134 subsequence. If the 4 were $n$, then there would be a 213 subsequence among the $A_{i}$. However, as the elements in $A_{i}, i>1$ are all arranged in decreasing order, the 213 must occur in $A_{1}$, contradicting condition 3 . Hence the $n$ can not be a 4 in a 2134 subsequence. Moreover, the above reasoning shows that there is no 213 subsequence to the left of $n$, so that any 4 must occur to the right of $n$.

Suppose that the 4 occurs within the $B_{i}, i<s$. Then because the elements of $B_{i}$, $i<s$, are arranged in decreasing order, the 2,1 , and 3 all occur in $C$ or the $A_{i}$. If the 1 is in the $A_{i}$, then so is the 2 , but then the 3 must lie in among the $B_{i}, i<s$, which is impossible, because the $B_{i}$ are strictly decreasing. So either the 2 occurs in the $A_{i}$ and the 12 occurs in $C$ or the 312 occurs in $C$. However, in either of these cases, we may take the 4 to be in $B_{s}$ and the 3 to be $a$. But condition 4 tells us that the subsequence $D$ formed by $a, B_{s}$, and $C$ avoids 2134 , so we have a contradiction, and the 4 cannot be among the $B_{i}, i<s$. 
If the 4 occurs in $B_{s}$, then the 213 occurs among $a_{s-1}, A_{s}$ and $C$. The 213 cannot occur in $A_{s} \cup a_{s-1}$, as noted above, and if both the 2 and the 1 occurred in $A_{s}$, then the 3 would be among the $B_{i}, i<s$, which is impossible, as $B_{i}>B_{s}$ for $i<s$. Thus, at most one element of $A_{s}$ is used, and we may as well take it to be $a$. But the subsequence $D$ formed by $a, B_{s}$, and $C$ avoids 2134 by condition 4 Finally, if the 4 occurs in $C$, then the whole subsequence lies in $C$, again contradicting condition 4 .

Now suppose that there were a 1243 subsequence in $\sigma$. If the 2 were to the left of $n$, the 1 would be also. But since the only possible increasing subsequences lie in $A_{1}$, the 4 must either be $n$ or lie in $A_{1}$. The former is impossible because there is nothing to the right of $n$ that could be larger than the 2 in $A_{1}$. In the latter case, both the 3 and 4 must lie in $A_{1}$, contradicting condition 3 , the statement that $A_{1}$ avoids 1243 . If the 2 is to the right of $n$, because the 2 and 4 form an increasing sequence, they must lie in $B_{s}$ or $C$. In either case, that would force the 1 to lie in $A_{s}$ or $C$. In the former case we could the the 1 to be $a$. Either way, we end up with a 1342 sequence in $D$, a contradiction.

\subsection{A Bijection Between $S_{n k}(1342,3124)$ and $S_{n k}(1243,2134)$}

We now state a lemma which will be proved later:

Lemma $7 S_{n k}(1342,312)=S_{n k}(1243,213)$.

Lemma 7 allows us to exhibit a bijection between $S_{n k}(1342,3124)$ and $S_{n k}(1243,2134)$. For $n \leq 3$ we can just map every permutation in $S_{n}$ to itself. Now choose some bijection between $S_{n k}(1342,312)$ and $S_{n k}(1243,213)$, which is possible by the lemma. Any permutation $\sigma$ in $S_{n k}(1342,3124)$ falls into one of Cases 1, 2, 3 or 4 above. If it falls into Case 1 , we have blocks $A_{1}>A_{2}>\cdots>A_{s}$ and $B_{1}>B_{2}>\cdots>B_{s}$, with $A_{i}$, $i>1$, and $B_{i}, i<s$ decreasing. We now permute the elements within block $A_{1}$ and the subsequence $D$. We have that $A_{1}$ avoids 1342 and 312 , and we can use the bijection between $S_{n^{\prime} k^{\prime}}(1243,213)$ and $S_{n^{\prime} k^{\prime}}(1342,312)$ for some $n^{\prime}, k^{\prime}$ to change it into a block that avoids 1243 and 213. Now note that the subsequence $D$ as described above avoids 1342 and 3124 and also has the property that the first number in $D$ is not the largest number in $D$. Then we can take the subsequence $D$ and use the bijection between $S_{n^{\prime \prime} k^{\prime \prime}}(1342,3124)$ and $S_{n^{\prime \prime} k^{\prime \prime}}(1243,2134)$ for some $n^{\prime \prime}, k^{\prime \prime}$ to turn $D$ into a subsequence that avoids 1243 and 2134 and has the property that the first number in $D$ is not the largest number in $D$. This is possible because, as we will see, our bijection respects whether or not a permutation in $S_{n}(1342,3124)$ or $S_{n}(1243,2134)$ begins with $n$ (in fact, it respects the position of $n$ in the permutation).

Finally, we can rearrange the blocks "vertically" by moving the smallest element in each $A_{i}$ down to the level of $A_{i+1}$, moving $B_{1}$ down to the level of $B_{2}$ and moving all but the the largest element of each $B_{i}, i>1$ down so that it lies between the smallest element of $A_{i}$ and the rest of $A_{i}$. Then we obtain a permutation $\tau$ in $S_{n}(1243,2134)$ by Propositions 5 and 6 . We can also go in the reverse direction-given a permutation $\tau$ in $S_{n}(1243,2134)$ that falls under Case 2 , we turn the $A_{i}$ and $B_{i}$ into blocks with $B_{i}>A_{i}>B_{i+1}$ and replace $A_{1}$ and $D$ by using the bijection between $S_{n^{\prime} k^{\prime}}(1243,213)$ 
and $S_{n^{\prime} k^{\prime}}(1342,312), S_{n^{\prime \prime} k^{\prime \prime}}(1342,3124)$ and $S_{n^{\prime \prime} k^{\prime \prime}}(1243,2134)$, respectively. Note that the sets $A_{i}, B_{i}, C$ and $a$ as defined above for $S_{n}(1342,3124)$ and $S_{n}(1243,2134)$ correspond to each other under this bijection [Figures 12.1, 16.1].

If $\sigma$ falls into Case 2, we have one block to the left of $n$. Then $A$ avoids 1342 and 312 . Then using the bijection between $S_{n^{\prime} k^{\prime}}(1243,213)$ and $S_{n^{\prime} k^{\prime}}(1342,312)$ for some $n^{\prime}$ and $k^{\prime}$ we can arrange that $A$ avoids 1243 and 213. Similarly, we can use the bijection between $S_{n^{\prime \prime} k^{\prime \prime}}(1342,3124)$ and $S_{n^{\prime \prime} k^{\prime \prime}}(1243,2134)$ for some $n^{\prime \prime}$ and $k^{\prime \prime}$ to transform $D$. Then we just need to move all the elements of $A$ except the smallest $a$ up so that they are larger than $B$ to obtain a permutation that avoids 1243 and 2134 and falls under Case 2 above [Figure 12.2, 16.2].

If $\sigma$ falls into Case 3, $B$ is empty. Thus, the elements to the left of $n$ avoid 1342 and 312, and $C$ avoids 1342 and 3124. Then we can turn $A$ into a sequence avoiding 1243 and 213 and turn $C$ into a sequence avoiding 1342 and 3124 using the above bijections. This results in a permutation that avoids 1243 and 2134 in Case 3 [Figure 12.3, 16.3].

Finally, in Case 4, we make use of the bijection between $S_{(n-1)(k-1)}(1342,3124)$ and $S_{(n-1)(k-1)}(1243,2134)$ to obtain a bijection between the permutations of length $n$ that begin with $n$ and begin with a decreasing sequence of length $k$. This completes the proof of Theorem 2.

We now give the proof of Lemma 7 .

Proof of Lemma 7: It is known that $\left|S_{n}(1342,312)\right|=\left|S_{n}(1243,213)\right|$ [8]. Now take any permutation $\sigma$ in $S_{n k}(1342,312)$. Then $\sigma$ begins with a decreasing sequence of length $k$. We claim that these $k$ elements are consecutive. Suppose otherwise, and let $a>b>c$ with $a$ and $c$ occurring consecutively among the first $k$ elements of $\sigma$. Then $b$ occurs later in $\sigma$ and $a c b$ is a 312 subsequence, a contradiction. Thus deleting the first $k-1$ elements, we obtain a permutation in $S_{(n-k+1)(1)}(1342,312)$. Moreover, given any permutation in $S_{(n-k+1)(1)}(1342,312)$, there is a unique way to add $k-1$ elements of the left of it to obtain a permutation in $S_{n k}(1342,312)$, namely by adding them so that the first $k$ elements form a decreasing sequence. Thus $\left|S_{n k}(1342,312)\right|=\left|S_{(n-k+1)(1)}(1342,312)\right|$.

Now take $\sigma$ in $S_{n k}(1243,213)$. We claim that the first $k-1$ elements are not only decreasing, but are consecutive and begin with $n$. Suppose otherwise, and let $a>b$ with $b$ occurring among the first $k-1$ elements of $\sigma$, but $a$ not occuring among the first $k-1$ elements of $\sigma$. Then let $c$ be the $k$-th element of $\sigma$, so that $a>b>c$. Since $a$ occurs later in $\sigma$ than $b$ and $c, b c a$ is a 213 subsequence, and we have a contradiction. Thus deleting the first $k-1$ elements, we obtain a permutation in $S_{(n-k+1)(1)}(1243,213)$. Moreover, given any permutation in $S_{(n-k+1)(1)}(1243,213)$, there is a unique way to add $k-1$ elements to the left of it to obtain a permutation in $S_{n k}(1342,312)$, namely by adding them so that the first $k-1$ elements are the largest elements in the permutation. Thus $\left|S_{n k}(1243,213)\right|=\left|S_{(n-k+1)(1)}(1243,213)\right|$. Using 


$$
\begin{aligned}
\left|S_{n}(1342,312)\right| & =\sum_{k=1}^{n}\left|S_{n k}(1342,312)\right|, \\
\left|S_{n}(1243,213)\right| & =\sum_{k=1}^{n}\left|S_{n k}(1243,213)\right|, \\
\left|S_{n k}(1342,312)\right| & =\left|S_{(n-k+1)(1)}(1342,312)\right|, \\
\left|S_{n k}(1243,213)\right| & =\left|S_{(n-k+1)(1)}(1243,213)\right|, \text { and } \\
\left|S_{n}(1342,312)\right| & =\left|S_{n}(1243,213)\right|,
\end{aligned}
$$

we can inductively show that $\left|S_{n 1}(1342,312)\right|=\left|S_{n 1}(1243,213)\right|$.

\subsection{Wilf Classes of Pairs in $S_{4}$}

It has previously been shown that

$$
\begin{aligned}
\left|S_{n}(1234,3214)\right| & =\left|S_{n}(4123,3214)\right|=\left|S_{n}(2341,2143)\right|=\left|S_{n}(1234,2143)\right|, \\
\left|S_{n}(1324,2143)\right| & =\left|S_{n}(1342,2431)\right|=\left|S_{n}(1342,2314)\right|=\left|S_{n}(1324,2413)\right| \\
& =\left|S_{n}(1342,3241)\right|, \\
\left|S_{n}(2413,3142)\right| & =\left|S_{n}(1234,2134)\right|=\left|S_{n}(1324,2314)\right|=\left|S_{n}(1342,2341)\right| \\
& =\left|S_{n}(3124,3214)\right|=\left|S_{n}(3142,3214)\right|=\left|S_{n}(3412,3421)\right| \\
& =\left|S_{n}(1324,2134)\right|=\left|S_{n}(3124,2314)\right|=\left|S_{n}(2134,3124)\right|
\end{aligned}
$$

The first set of Wilf-equivalences above was shown in [4], the second in [1], [6], and the third in [3], [7], [2]. Numerical evidence demonstrates that the only other Wilfequivalences for pairs of permutations of length 4 are $\left|S_{n}(1342,2143)\right|=\left|S_{n}(3142,2341)\right|$ and $\left|S_{n}(1342,3124)\right|=\left|S_{n}(1243,2134)\right|$, thus completing the classification of Wilf classes

for pairs of permutations of length four. Finally, it is interesting to note that writing the first equivalence as $\left|S_{n}(2143,3241)\right|=\left|S_{n}(3142,2341)\right|$, both of the Wilf-equivalences shown in this paper arise from interchanging the 2 and the 3 in each pair of permutations. It would be interesting to consider whether this is evidence of a more general pattern.

\section{Acknowledgements}

This work was done at the University of Minnesota, Duluth under the supervision of Joseph A. Gallian. Many thanks to Joe for his encouragement and support, Philip Matchett for reading drafts and providing copious comments, Geir Helleloid for listening to my ideas, Eric Egge for suggesting this problem, and Reid Barton for providing me with numerical evidence. This research was supported by NSF grant DMS-0137611 and NSA grant H-98230-04-1-0050. 


\section{References}

[1] Bóna, Miklós. The permutation classes equinumerous to the smooth class. Electronic Journal of Combinatorics 5 (1998), \#R31.

[2] Gire, Sophie. Arbres, permutations motifs exclus et cartes planaires: quelques problmes algorithmiques et combinatoires, Thse de l'Universit de Bordeaux I (1993).

[3] Kremer, Darla. Permutations with forbidden sequences and a generalized Schroeder number. Discrete Mathematics 218 (2000), 121-130.

[4] Kremer, Darla and Wai Chee Shiu. Finite transition matrices for permutations avoiding pairs of length four patterns. Discrete Mathematics 268 (2003), 171-183.

[5] Simion, Rodica and Frank W. Schmidt. Restricted permutations. European Journal of Combinatorics 6 (1984), 383-495.

[6] Stankova, Zvezdelina. Forbidden sequences. Discrete Mathematics 132 (1994), 291316.

[7] West, Julian. Permutation trees and the Catalan and Schröder numbers. (from http://www.mala.bc.ca/ ${ }^{\sim}$ westj/papers.htm)

[8] West, Julian. Generating trees and forbidden sequences. (from http://www.mala.bc.ca/ ${ }^{\sim}$ westj/papers.htm) 